\title{
The solid-state fermentation of Artemisia capillaris leaves with Ganoderma lucidum enhances the anti-inflammatory effects in a model of atopic dermatitis
}

\author{
HYEONG-U SON ${ }^{1}$, SEUL LEE ${ }^{1}$, JIN-CHUL HEO ${ }^{2}$ and SANG-HAN LEE ${ }^{1}$ \\ ${ }^{1}$ Department of Food Science and Biotechnology, Kyungpook National University, Daegu 41566; \\ ${ }^{2}$ Department of Medical Genomics, Keimyung University School of Medicine, Daegu 41931, Republic of Korea
}

Received November 3, 2015; Accepted March 23, 2017

DOI: 10.3892/ijmm.2017.2945

\begin{abstract}
Artemisia capillaris, which belongs to the Asteraceae family and the genus Artemisia, has been reported to exert inhibitory effects on diabetes, cancer and inflammation. In this study, in order to enhance the bioactivity potential of the leaves of Artemisia by Ganoderma lucidum mycelium, we prepared aqueous samples of Artemisia capillaris (Ac) leaves, Ganoderma lucidum $(G l)$ and aqueous fractions produced by the solid fermentation of Ganoderma lucidum on Artemisia capillaris leaves $(a f A c / G l)$. Thereafter, we evaluated whether these samples have potential to attenuate inflammation-related symptoms in an amimal model of 2,4-dinitrofluorobenzene (DNFB)-induced atopic dermatitis. We found that $a f A c / G l$ exhibited enhanced anti-inflamamatory activity following the solid fermentation process when compared with $A c$ or $G l$ on ear thickness, ear epidermal thickness and eosinophil infiltration in the skin tissues. The expression of nitric oxide (NO) synthases (NOSs) was measured by immunohistochemical staining. The results revealed that $a f A c / G l$ decreased endothelial NOS and inducible NOS expression compared with the DNFB group, while neuronal NOS expression was not altered. By comparing NO production, we found that as opposed to $A c, a f A c / G l$ has potential to inhibit atopic dermatitis-related symptoms during the inflammatory event. As regards matrix metalloproteinase (MMP) expression patterns, afAc/Gl exerted potent inhibitory activity on the mRNA expression of MMP-2, -7, -9, -12, -14 and -19. Taken together, these results suggest that the solid state fermentation of $A c$ by $G l$ is an effective strategy to obtaining useful ingredients which are converted into valuable compounds during an atopic inflammatory insult.
\end{abstract}

Correspondence to: Dr Sang-Han Lee, Department of Food Science and Biotechnology, Kyungpook National University, 80 Daehak-ro, Buk-gu, Daegu 41566, Republic of Korea

E-mail: sang@knu.ac.kr

Key words: Artemisia capillaris, enhanced anti-inflammatory potential, atopic dermatitis, solid fermentation, Ganoderma lucidum

\section{Introduction}

Atopic dermatitis is a skin disease with inflammatory, pruritic, chronic and relapsing symptoms and up to $20 \%$ of children are affected worldwide (1). Atopic dermatitis is caused by unbalanced Th cells as Th2 cykokine increase, and is characterized by increased serum levels of IgE and peripheral eosinophilia (2). Atopic dermatitis is well known as a chronic inflammatory disease, as it is caused by defective skin barrier function (3) and by the overexpression of inflammatory factors, such as cyclooxygenase-2 (COX-2) and inducible nitric oxide (NO) synthase (iNOS) (4). Inflammatory processes are mediated through COX-2 and iNOS which generate NO and prostaglandin $\mathrm{E}_{2}$ (5). Macrophages, generally stimulated by lipopolysaccharide (LPS), also play major role in inflammation as a self-defense against innate immunity (6).

Artemisia capillaris (A. capillaris; Ac) belongs to the family of Asteraceae and the genus Artemisia, which has been traditionally used as a medicinal herb and as a hepatoprotective, analgesic and antipyretic agent in Asia (7). It also has been reported to have various functions against inflammation (6), cancer (8), Helicobacter pylori infection (9) and hepatotoxicity (10). Recently, it was reported that $70 \%$ ethanol extract of $A c$ exerted inhibitory effects on atopic dermatitis-like skin lesions via the downregulation of serum histamine content and IgE expression (11). In our laboratory, we have been eager to screen out useful plant resources for industrial purposes. In order to improve the bioavailability of natural ingredients, the fermentation technique had been used to enhance their activity, as well as to reduce their toxicity. Even though submerged fermentation is a more common process in microbial production, solid fermentation is more effective as regards productivity and bioconversion (12). Moreover, it has been well documented that solid fermentation is a simple, easy and economical process which requires limited facilities; however, it is a timeconsuming process compared to liquid fermentation. It has been previously demonstrated that solid state fermented black bean by Aspergillus species (13), and solid fermented wheat grain by Grifola species enhanced the antioxidant activity of black bean $(13,14)$. Chickpea, which is a type of bean, has also been shown to exert anti-hyperglycemic effects following solid-state bioconversion by Rhizopus oligosporus (15). These are good 
examples that non-degradable biomass can be converted into valuable biomaterials by solid fermentation using fungi, yeast and bacteria, which can enhance the bioavailability of natural resources.

The present study demonstrated that $A c$, following solid state fermentation with Ganoderma lucidum (G. lucidum; $G l$ ), had the potent potential to inhibit atopic dermatitis-related symptoms in an animal model of 2,4-dinitrofluorobenzene (DNFB)-induced atopic dermatitis. To the best of our knowledge, there are no available studies to date examining the effects of $A c$ following solid fermentation on atopic dermatitis. Solid fermentation is considered an innovative process with good pharmaceutical potential as the antioxidant capacity of $A c$ increases following solid fermentation with various mycelia. In this study, we focused on $G l$, which has great potential to improve the antioxidant capacity, in order to investigate whether $A c$ has the potential to alleviate inflammation-related symptoms before and after solid fermentation.

We hypothesized that following the solid fermentation $A c$ leaves with $G l$ will result in the production of active compounds which may have the potential to enhance the anti-inflammatory effects of $A c$ on atopic dermatitis.

\section{Materials and methods}

Materials and reagents. DNFB (Sigma, St. Louis, MO, USA; dissolved in polyethylene glycol) was sterile filtered using a syringe filter $0.45 \mu \mathrm{m}$ (Pall Life Sciences, Port Washington, NY, USA) and used for the induction of atopic dermatitis in mice. Polyethylene glycol (P3265; Sigma) was used as a vehicle for application on the mouse ear surface.

Animal care. C57BL/6 mice (6 weeks of age, male, weighing between 20 and 23 g, 5 mice per group) were purchased from Samtaco Korea (Osan, Korea). The mice were allowed to acclimatize for 7 days in an air-ventilated animal room at a temperature of $22+1^{\circ} \mathrm{C}$ and a humidity of $65+5 \%$ under a 12-h light/dark cycle. All animal experiments were carried out according to the guidelines of the Committee of the International Association for the Study of pain Research and Ethical Issues (16) and following the approval (permission no. KNU-2014-0145) of the KNU Animal Ethics Committee (Chair, Dr Hee-Kyung Jin).

Solid fermentation of Gl on Ac leaves. The plant was obtained from a local supplier at Andong, Korea and $G l$ was donated from Farmbios Co., Ltd. (Daegu, Korea). Fermentation was carried out as previously described by Shin et al (17), with a slight modification. In brief, the mycelia of $G l$ was cultured on potato dextrose broth (Difco Co., Detroit, MI, USA) at $25^{\circ} \mathrm{C}$ in a shaking incubator for 20 days. A. capillaris was dried and autoclaved at $121^{\circ} \mathrm{C}$ for $20 \mathrm{~min}$ (Fig. 1A), and $10 \mathrm{~g}$ of the grown mycelia with potato dextrose agar medium was then adapted into $200 \mathrm{~g}$ of $A$. capillaris. Even though the mycelia were in liquid potato dextrose agar medium, they were rapidly dried ( $<5 \%$ moisture) and the mixed materials were subjected to solid fermentation. This fermentation was performed at $25^{\circ} \mathrm{C}$ for 2 weeks, and we finally observed the white fruit body of G. lucidum (Fig. 1B). The fermented product was extracted with distilled water ina shaking incubator in at $25^{\circ} \mathrm{C}$ for $24 \mathrm{~h}$, and it was then lyophilized (MCTD85; Il-Shin,
Gyeonggi-do, Korea) following filtration. The voucher specimens of the plant and fermented samples have been deposited in the Laboratory of Enzyme Biotechnology, Kyungpook National University, Daegu, Korea.

Animal model of DNFB-induced atopic dermatitis. We created an animal model of DNFB-induced atopic dermatitis as previously described, with some modifications $(18,19)$. In brief, $50 \mu \mathrm{l}$ of $0.5 \%$ (w/v) DNFB solution (Sigma; dissolved in polyethylene glycol) was applied on the abdominal skin of mice for primary sensitization. At 5 days following sensitization, $20 \mu 10.2 \%$ (w/v) DNFB was applied to the ear skin at 3-day intervals. Treatment with $20 \mu \mathrm{l}$ of the samples (final concentration, $100 \mu \mathrm{g} / \mathrm{ml}$ ) was carried out 1 day following exposure to DNFB (Fig. 1C). In total, DNFB and the treatment samples were applied 4 times and we observed inflammation-related symptoms, such as swelling or scabs on the mouse ear surface. At 2 days following the final sample application, the mice were sacrificed and the ear organs were isolated. The experimental groups were divided into the NT (vehicle alone) group, DNFB (DNFB treatment alone) group, $A c$ (DNFB treatment and thereafter application of aqueous fraction of $A c$ ) group, $G l$ (DNFB treatment and thereafter application of aqueous fraction of $G l$ ) group and afAC/Gl (DNFB treatment and thereafter application of aqueous fraction produced by solid fermentation of $G l$ on $A c$ ) group, as described in Fig. 1.

Hematoxyline and eosin $(H \& E)$ staining and immunohistochemistry. H\&E staining was carried out as previously described (20). In brief, the ear tissues were entirely cut and fixed with $10 \%$ formalin solution in phosphate-buffered saline (PBS) for at least $12 \mathrm{~h}$. In order to maintain the linear shape of the ear tissue, it was placed between 2 coverslips following formalin fixation. The organ sections were fixed in paraffin lengthwise and transferred onto slides. Subsequently, $3 \%$ hydrogen peroxide in methanol solution was applied to prevent endogenous peroxidase activity and the slides were stained with H\&E (HHS32 and HT110332' Sigma). To perform immunohistochemical analysis, each slide was treated with a $10 \%$ normal goat serum for $1 \mathrm{~h}$, and the slides were then incubated overnight at $4{ }^{\circ} \mathrm{C}$ with rabbit anti-mouse antibodies against iNOS (ab15326), endothelial NOS (eNOS; ab5589) and neuronal NOS (nNOS; ab72428) (all from Abcam, Cambridge, MA, USA), as previously described (21). To observe the immunohistochemical sections, a Nikon microscope and camera (Eclipse, TE-2000U; Nikon, Tokyo, Japan) were used.

Evaluation of atopic dermatitis-related symptoms. Various factors of atopic responses (ear swelling, ear epidermal swelling, total cell count and eosinophil count) were measured on the H\&E-stained sections. First, ear thickness or ear epidermal thickness was calculated using ImageJ software [1.48 version, National Institutes of Health (NIH)]. Next, total cell and eosinophil counts were determined. Due to the of unique morphology of eosinophils, total cells were counted first, and then eosinophil cells were observantly counted under a microscope (Eclipse, TE-2000U; Nikon) at x400 magnification.

Measurement of NO levels and cytotoxicity assay. NO production and cell viability were assayed using the RAW264.7 cell line, 
A

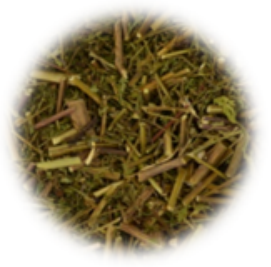

C

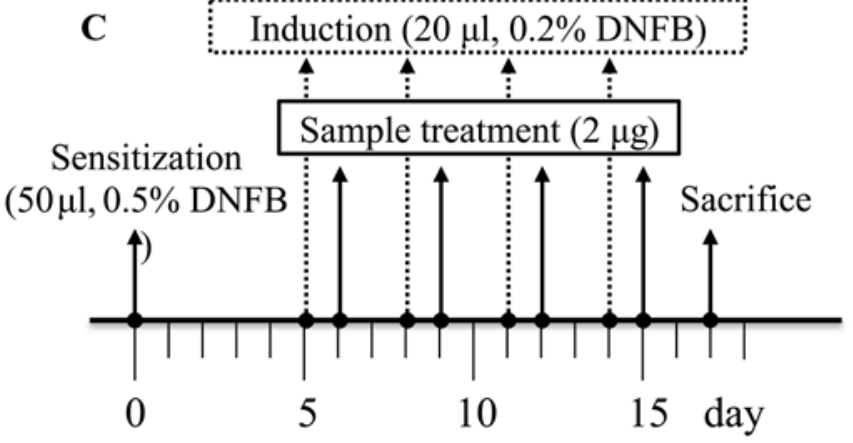

Figure 1. Classical images of a product of solid fermentation of Artemisia capillaris by Ganoderma lucidum, and experimental schedule. (A) Before and (B) after fermentation with G. lucidium. (C) Experimental schedule in our mouse model of 2,4-dinitrofluorobenzene (DNFB)-induced atopic dermatitis.

which was obtained from American Type Culture Collection (Cat. no. TIB-71; Manassas, VA, USA). The cells were seeded as $5 \times 10^{4}$ cells/well with $200 \mu$ l of medium [ $10 \%$ fetal bovine serum (FBS; Hyclone, Lagan, UT, USA) in Dulbecco's modified Eagle's medium (DMEM; Hyclone)] in a 96-well plate. The cells were pre-incubated in a 96-well plate for $4 \mathrm{~h}$, and this was followed by the addition of lipopolysaccharide (LPS, $1 \mathrm{mg} / \mathrm{ml}, \mathrm{L} 2880$; Sigma) and the treatment samples and incubation at $5 \% \mathrm{CO}_{2}$, $37^{\circ} \mathrm{C}$ for $24 \mathrm{~h}$. To detect NO production, $100 \mu \mathrm{l}$ of supernatants from the cell culture plate were moved to a new 96-well plate, and $100 \mu \mathrm{l}$ of Griess reagent (G4410; Sigma) were then added. According to the reaction between NO product and Griess reagent, the color changed yellow to pink and the absorbance was then measured at $520 \mathrm{~nm}$ on a spectrophotometer (VICTOR3; Perkin Elmer, Wellesley, MA, USA). To measure the cytotoxicity of the treatment samples, cell culture medium was removed from a 96-well cell culture plate which had remained following NO assay. Subsequently, $100 \mu \mathrm{l}$ of DMEM with $0.5 \mathrm{mg} / \mathrm{ml}$ methylthiazolyldiphenyl-tetrazolium bromide (MTT, M2128) solution were added followed by incubation for $30 \mathrm{~min}$. The supernatants were removed and the plates were washed twice with PBS. Thereafter, the produced formazan crystals from the MTT solution were dissolved into DMSO and cell viability was assessed by measuring the absorbance at $595 \mathrm{~nm}$ on a spectrophotometer.

Reverse transcription-polymerase chain reaction (PCR) for $m R N A$ expression. To determine the effects of $A$. capillaris on mRNA expression in RAW264.7 cells, the cells were seeded at $5 \times 10^{6}$ cells/well in a 6 -well plate (BD Falcon, NJ, USA). The cells were treated with LPS $(1 \mu \mathrm{g} / \mathrm{ml})$ for $4 \mathrm{~h}$ and with the sample extracts at $30 \mu \mathrm{g} / \mathrm{ml}$ for $5 \mathrm{~h}$. Following the removal of the cell supernatant, RNA was isolated using TRIzol ${ }^{\circledR}$ reagent (Invitrogen, New York, NY, USA). Total RNA was reverse transcribed into cDNA using RT-PCR master mix (Qiagen,
Valencia, CA, USA). cDNA was amplified with manufactured primers as follows: matrix metalloproteinase (MMP)-2 forward, ACCAGAACACCATCGAGACC and reverse, AAA GCATCATCCACGGTTTC; MMP-7 forward, GAGTGCC AGATGTTGCAGAA and reverse, CCATCAAAGGGGAA GCTGT; MMP-9 forward, GGGTTTCTGTCCAGACCAAG and reverse, GGATGCCGTCTATGTCGTCT; MMP-12 forward, TTGATGGCAAAGGTGGTACA and reverse, CGAAATGTGCTGGGGTTAAG; MMP-14 forward, CCGCCATGCAAAAGTTCTAT and reverse, GCCTTGAT CTCAGTCCCAAA, MMP-19 forward, GACATTCGCCT CTCTTTCCA and reverse, AGGTCCCCTCAGTCCAGAGT; and glyceraldehyde 3-phosphate dehydrogenase (GAPDH) forward, ATGTTCCAGTATGACTCCAC and reverse, GCCAAAGTTGTCATGGATGA (Bioneer, Daejeon, Korea) and detected using $1 \%$ agarose gel by ChemiDOC ${ }^{\text {TM }} \mathrm{XRS}^{+}$ Molecular Imager (Bio-Rad, Hercules, CA, USA). The band density from the esults of the PCR product was calculated using Image Lab software (5.0 version; Bio-Rad).

High-performance liquid chromatography photodiode array (HPLC-PDA) analysis. Cholorogenic acid and caffeic acid were analyzed as described in a previous study (11). HPLC analysis was performed using a Waters 2695 HPLC system (Waters Co., Milford, MA, USA) fitted with a binary pump, an autosampler, a column oven and a PDA detector. The HPLC system was monitored by a computer equipped with Empower software (Waters Co.). Chromatographic separation was performed on a HYPERSIL ODS C18 column $(250 \times 4.6 \mathrm{~mm}$, $5 \mu \mathrm{m}$; Thermo Scientific, Waltham, MA, USA). The chromatographic binary mobile phase consisted of $1 \%(\mathrm{v} / \mathrm{v})$ aqueous acetic acid (A) and 1\% (v/v) acetic acid in acetonitrile (B) for chlorogenic acid analysis. The gradient flow rates were as follows: $0-5 \mathrm{~min}, 0-10 \% \mathrm{~B}$; 5-30 $\mathrm{min}, 10-50 \% \mathrm{~B} ; 30-35 \mathrm{~min}$, $50-50 \% \mathrm{~B} ; 35-40 \mathrm{~min}, 50-10 \% \mathrm{~B}$. The flow rate and injection volume were $1.0 \mathrm{ml} / \mathrm{min}$ and $10 \mu \mathrm{l}$, respectively. The detection wavelength was set at $320 \mathrm{~nm}$. Caffeic acid separated at a flow rate of $0.5 \mathrm{ml} / \mathrm{min}$; solvent $\mathrm{A}$ was acetonitrile and solvent $\mathrm{B}$ was acetic acid solution (dilute $20 \mathrm{ml}$ of glacial acetic acid to $1,000 \mathrm{ml}$ with DI water, $\mathrm{pH}$ 2.6). Elution was performed according to the following conditions: $0-5 \mathrm{~min}, 5 \% \mathrm{~A} ; 25-30 \mathrm{~min}, 35 \% \mathrm{~A}$; 35-40 $\min 90 \% \mathrm{~A} ; 40.1-41 \mathrm{~min}, 5 \% \mathrm{~A}$ and monitored at $280 \mathrm{~nm}$.

Statistical analysis. Data are expressed as the means \pm standard deviation. Statistical significance was determined by one-way ANOVA with Tukey's post-hoc test using the SPSS 21.0 program (SPSS, Inc., Chicago, IL, USA). The critical level for significance was set at $\mathrm{P}<0.05$.

\section{Results and Discussion}

Effects of aqueous fraction produced by the solid fermentation of Gl on Ac (afAc/Gl) on a mouse model of DNFB-induced atopic dermatitis. To determine the inhibitory effects of afAc/Gl against atopic dermatitis-related symptoms, we established a mouse model of DNFB-induced atopic dermatitis (Fig. 1C) and analyzed the data. First, we conducted an observation of the ear surface, as atopic dermatitis is characterized by the appearance of inflammation. In the DNFB-treated mice, due to scratching of the ears, we found that the skin had swelled and scabs 


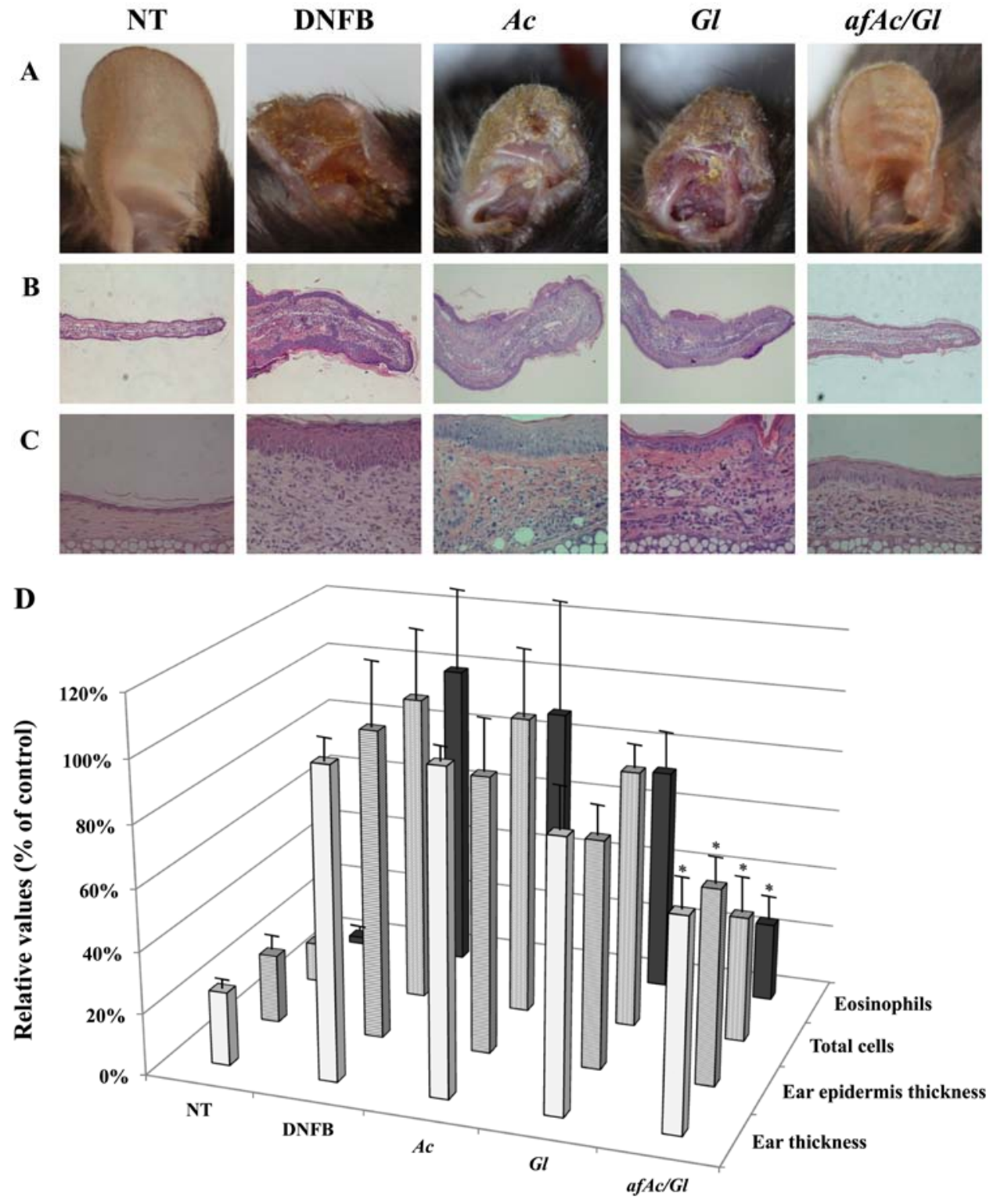

Figure 2. Inhibitory effect of an aqueous extract of Artemisia capillaris following fermentation with Ganoderma lucidum against 2,4-dinitrofluorobenzene (DNFB)-induced atopic dermatitis-related symptoms in C57BL/6 mice. Apart from the NT group, all groups were treated $0.2 \%$ (w/v) DNFB on the ear surface (concentration of $30 \mu \mathrm{g} / \mathrm{ml}$ ). NT, no treatment group; Ac, Artemisia capillaris; Gl, Ganoderma lucidum; afAc/Gl, aqueous fraction produced by solid fermentation of Ganoderma lucidum on Artemisia capillaris. (A) Images of ears of mice. (B and C) Observation of atopic dermatitis-related symptoms by H\&E staining, (B) x40 magnification and (C) x400 magnification. (D) Assessment of atopic dermatitis-related symptoms reduction by afAc/Gl. Ear thickness (first row), ear epidermal thickness (second row), and total cell (third row), eosinophil (forth row) counts were assessed as described in the Materials and methods. Data were randomly extracted 5 times from each paraffin-embedded section, and calculated using ImageJ software. The values of quantitative measurement were adjusted to those of the DNFB-treated group. Data are expressed as the means \pm standard deviations $(\mathrm{n}=5)$; ${ }^{*} \mathrm{P}<0.05$.

had formed on the DNFB-applied tissue sites. However, we found that the sample-treated mice, particularly those treated with afAc/Gl, exhibited less scratching behavior, ear swelling and scabs. At the end of the experimental period, the ears of the mice in the DNFB-treated group had become twisted in appearance due to the inflammatory response in the body, whereas in the sample-treated groups, the ears of the mice were only moderately twisted in appearance; in particular, the ears of the mice in the $a f A c / G l$-treated group were almost similar in appearance to those of the control group (Fig. 2A). To obtain more reliable positive data, we assessed ear thickness on H\&E-stained slides using ImageJ software. The paraffinembedded ear tissues exhibited incremental thickness form the edge of the outer site of the ear towards the inside; therefore, we selected the same section and averaged the length for further investigation. The whole appearance of the paraffin-embedded ear tissue was observed under a microscope at a magnification of $\mathrm{x} 40$ (Fig. 2B), and half the ear tissue on cartilage was observed at $x 400$ magnification (Fig. 2C). The results revealed that ear thickness in the afAc/Gl-treated mice decreased by $32 \%$, while that of the $\mathrm{Gl}$-treated mice decreased by $13 \%$; ear thickness in the $A c$-treated mice did not differ compared to the NT group (Fig. 2D). In addition, ear epidermal thickness in the afAc/Gl-treated mice decreased by $37 \%$, which was the most signicant among the sample treatment groups.

In atopic dermatitis, eosinophil numbers depend on various factors, such as platelet-activating factor (PAF), cytokines, leucotrienes and prostanoids (22). Therefore, eosinophil 
A
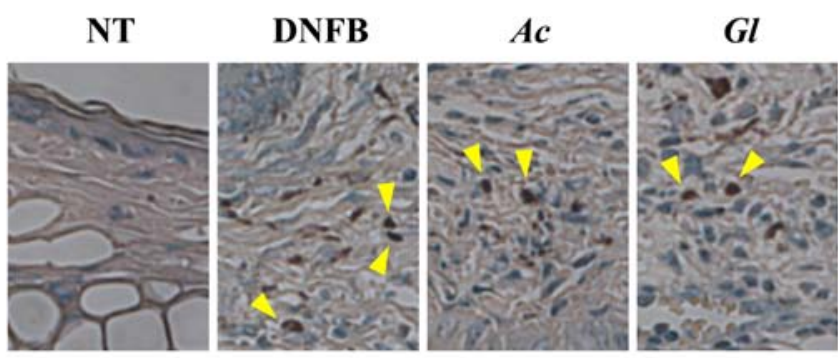

af $A c / G l$

B

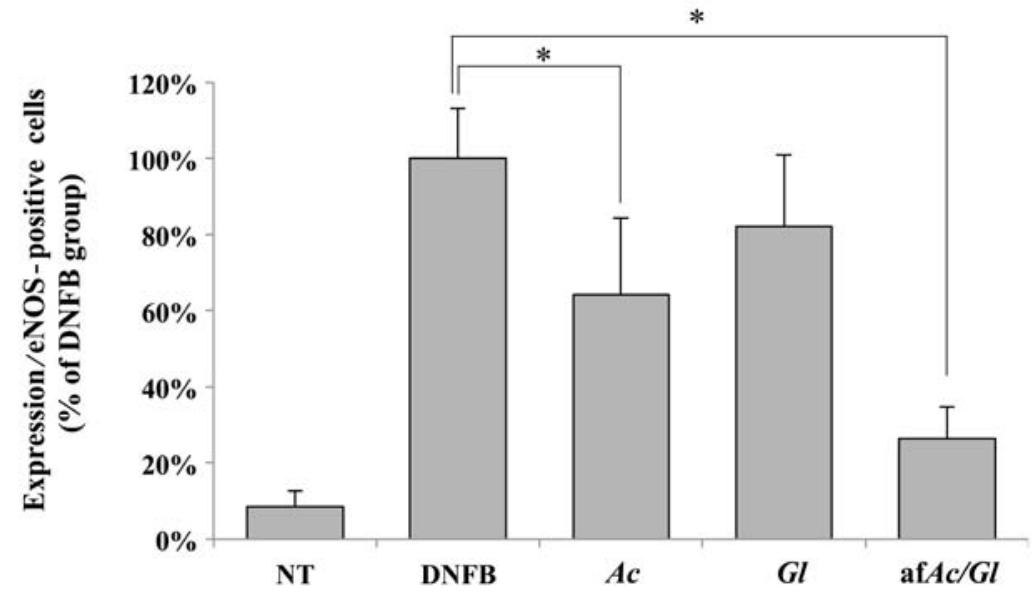

C
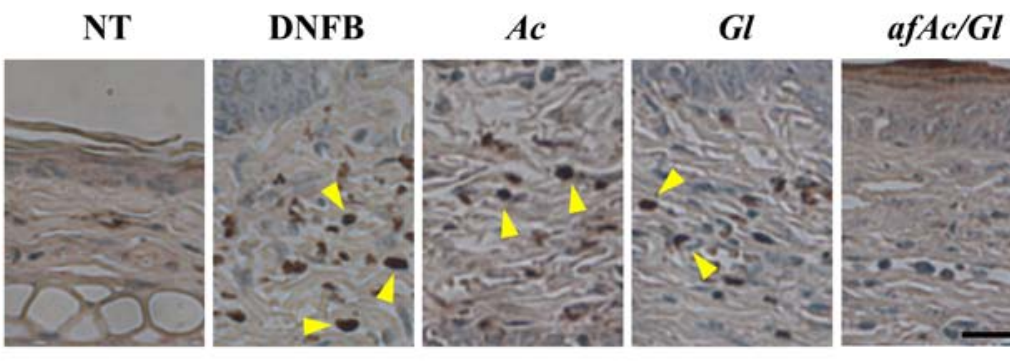

D

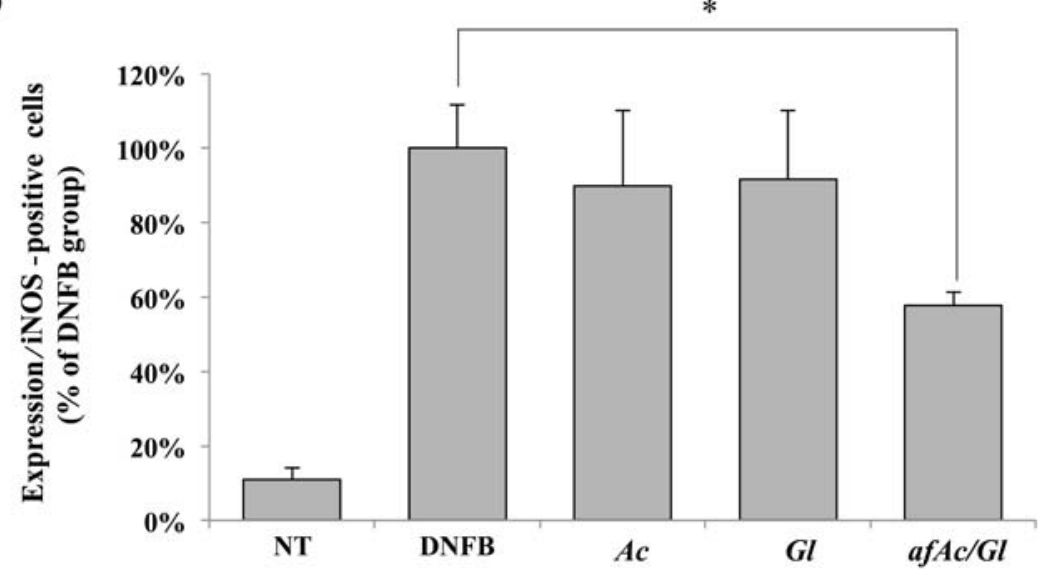

Figure 3. Inhibition of endothelial nitric oxide synthases (eNOS) expression with or without the application of the aqueous fraction produced by solid fermentation of Ganoderma lucidum on Artemisia capillaris $(\mathrm{afAc} / \mathrm{Gl})$. Expression and number of postive cells for of (A and B) eNOS and (C and D) inducible NOS in 2,4-dinitrofluorobenzene (DNFB)-treated mice assayed as described in the Materials and methods. Scale bar, $25 \mu \mathrm{m} .{ }^{*} \mathrm{P}<0.05$.

numbers can be predicted by increased itching, which may not be observed in normal skin. As shown by our results, eosinophils were observed at limited numbers in the control group; however, the eosinophil numbers were markedly inreased in the DNFB-treated group. Treatment with afAc/Gl decreased the number of eosinophils by approximately $74 \%$ compared to those of the DNFB-treated group (Fig. 2D). These results suggest that the solid fermentation of $a f A c$ by $G l$ may be used to enhance the anti-inflammatory potential in atopic dermatitis.

afAc/Gl extract exhibits enhanced anti-inflammatory potential in atopic dermatitis by reducing eNOS expression in vivo. We then investigated whether eNOS plays a pivotal role in downregulating the protein expression in skin tissues exposed to DNFB. 

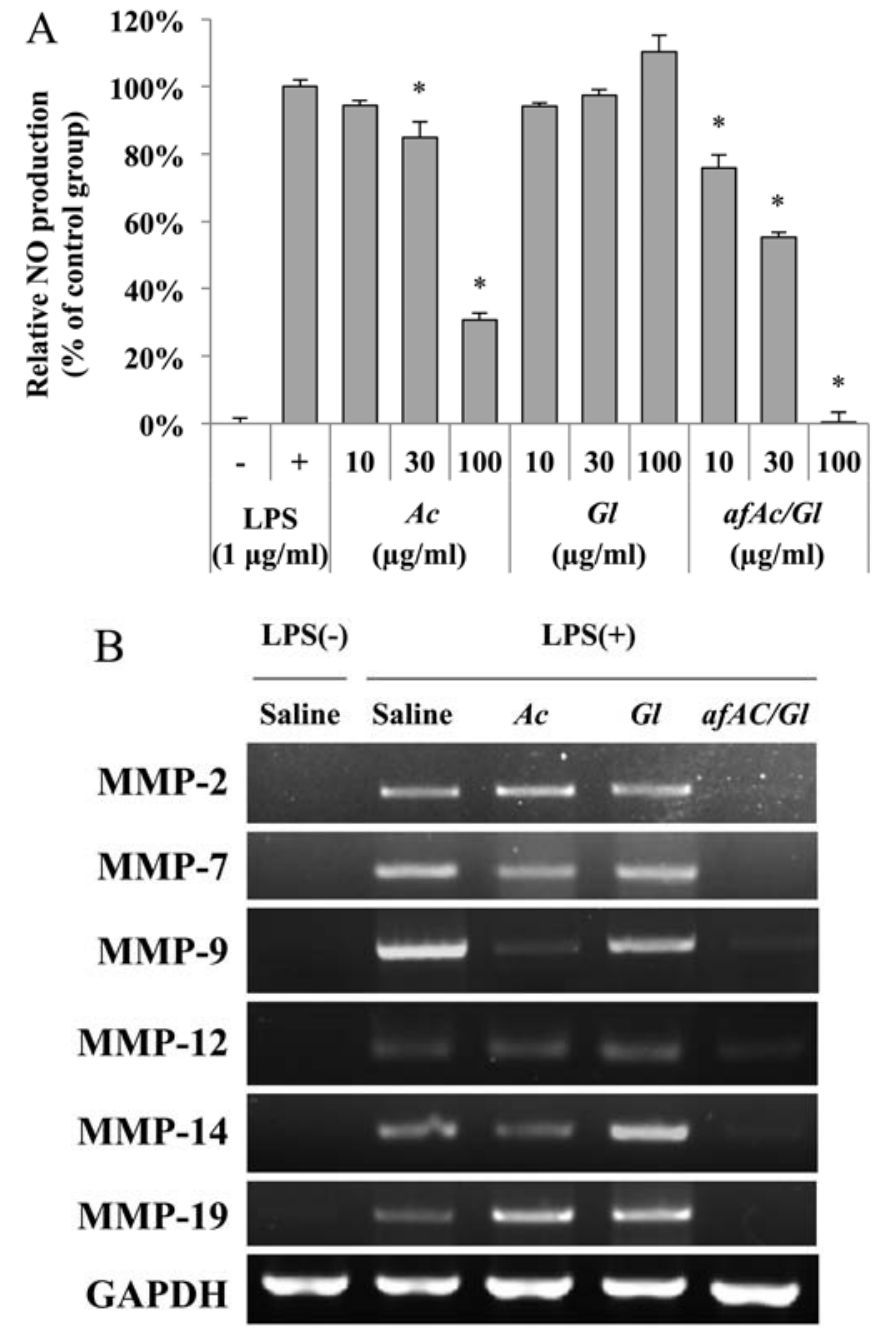

Figure 4. Inhibitory effect of nitric oxide (NO) production in RAW264.7 cells. (A) Inhibitory effect of NO production by aqueous fraction produced by solid fermentation of Ganoderma lucidum on Artemisia capillaris (afAc/Gl) in RAW264.7 cells. Cells were seeded at a concentration of $5 \times 10^{4}$ cells/well in a 96-well plate. The cells were exposed to LPS and treated with the samples and incubated for $24 \mathrm{~h}$. Griess reagent was used for the measurement of NO. (B) Inhibition of matrix metalloproteinase (MMP) expression in RAW264.7 cells by afAC/Gl. RAW264.7 cells were treated with sample extract $(10 \mu \mathrm{g} / \mathrm{ml})$ for $5 \mathrm{~h}$, and then exposed to LPS $(1 \mu \mathrm{g} / \mathrm{ml})$ for $4 \mathrm{~h}$. Relative intensity of the PCR band was calculated as described in the Materials and methods.

Protein expression was determined by immunohistochemical microscopic analysis at a magnification of x400 (Fig. 3A). We first counted the number of eNOS-positive cells on overall paraffin-embedded ear tissues. The results revealed that the tissue of the afAc/Gl-treated mice did not exhibit any eNOSpositive cells, as opposed to the tissue of the DNFB-, $A c$ - or $G l$-treated mice (Fig. 3A, compare the number of arrowheads). Thus, eNOS expression was increased by DNFB and $a f A c / G l$ treatment attenuated this effect. As shown in Fig. 3B, the number of eNOS-positive cells in the afAc/Gl-treated group was significantly decreased compared to the DNFB group. Treatment with $A c$ also decreased the numer of eNOS-positive cells, but not as significantly as $a f A c / G l$. Similarly, iNOS was also excessively expressed in the DNFB-treated group (Fig. 3C and D). However, only treatment with $a f A c / G l$ significantly decreased the number of iNOS-positive cells. The expression of iNOS did not exhibit any significant changes between the groups (data not shown).
NO is an important molecule in body homeostasis as a secondary signal mediator, and it is synthesized from L-arginine by NOS [nNOS (NOS1), iNOS (NOS2) and eNOS (NOS3)]. iNOS is mainly well-known as an inflammatory mediator with a function similar to COX-2, which is generally upregulated in inflammation (23-25). It has been well-documented that iNOS is upregulated during the inflammatory response in various cancer cells exposed to LPS, while nNOS (NOS1) and eNOS (NOS3) are expressed constitutively (23). iNOS is a key enzyme among the NOSs, although the three NOSs have been shown to be involved in various inflammation-related diseases (26). Recently, it was demonstrated that, in dogs with D. farinae-induced atopic dermatitis, eNOS was strongly expressed along with iNOS, NADPH oxidase 4 (NOX4), transforming growth factor- $\beta 1$ (TGF- $\beta 1$ ), COX-2 and myeloperoxidase (MPO), suggesting that these molecules are crossly associated with the cascade for the metabolic pathway (27). It has been reported that eNOS and iNOS are expressed in skin biopsies of patients with atopic dermatitis (28). On the other hand, in another study on colitis, eNOS-transgenic mice exhibit lower weight loss, less mortality, less inflammationinduced damage, les colonic myeloperoxidase activity and a lower expression of tumor necrosis factor- $\alpha$ compared to WT mice (29). Therefore, the study of eNOS expression has yielded conflicting results; however, there seems to be a lower expression of eNOS with a less active inflammatory response, which are taken together with our data on Fig. 3B.

Asthma is very similar to atopic dermatitis by comparison of the molecular mechanisms and symptoms. In a previous study, NOS-deficient mice were treated with ovalbumin to induce airway inflammation, and thereafter airway hyperresponsiveness was evaluated using methacholine response. In that study, the iNOS-deficient mice exhibited similar results to the WT mice, but the nNOS-deficient mice exhibited significantly less airway responsiveness than the WT mice (30). Overall, these data suggest that NOSs are major factors inatopic dermatitis; we confirmed that eNOS, as opposed to iNOS, was a more critical controlling factor in atopic dermatitis-related symptoms.

afAc/Gl downregulates MMP expression in vitro. We then also used RAW264.7 cells to prove which molecular target(s) is regulated by the fraction. Anti-inflammatory effects can be examined by NO production, which was induced by LPS (500 ng/ml). As a result, no toxicity was observed from all samples by the MTT assay. The afAc/Gl (most potent inhibitory activity at $100 \mu \mathrm{g} / \mathrm{ml}$ ) inhibited NO activity more significantly compared with Ac (69\% inhibitory activity), although $G l$ had no significant effect (Fig. 4A, compare bars 6-9).

MMPs are also a family of zinc-dependent enzymes that regulate the extracellular matrix as key factors in inflammation and disease (31). We found that $a f A c / G l$ extract regulated the expression of MMP-2, -7, -9, -12, -14 and -19 in RAW264.7 cells; however, $A c$ had a lesser effect on MMP expression compared toafAc/Gl, even though $A c$ significantly decreased MMP-9 expression (Fig. 4B). MMP-1a-MMP-29 have been shown to be expressed in various cell lines, indicating that these proteases are associated with inflammatory events in various cell populations. Among these, MMP-2 and -9 have been shown to be associated with allergic inflammation (32). A previous study demonstrated that MMP-2 was expressed in 

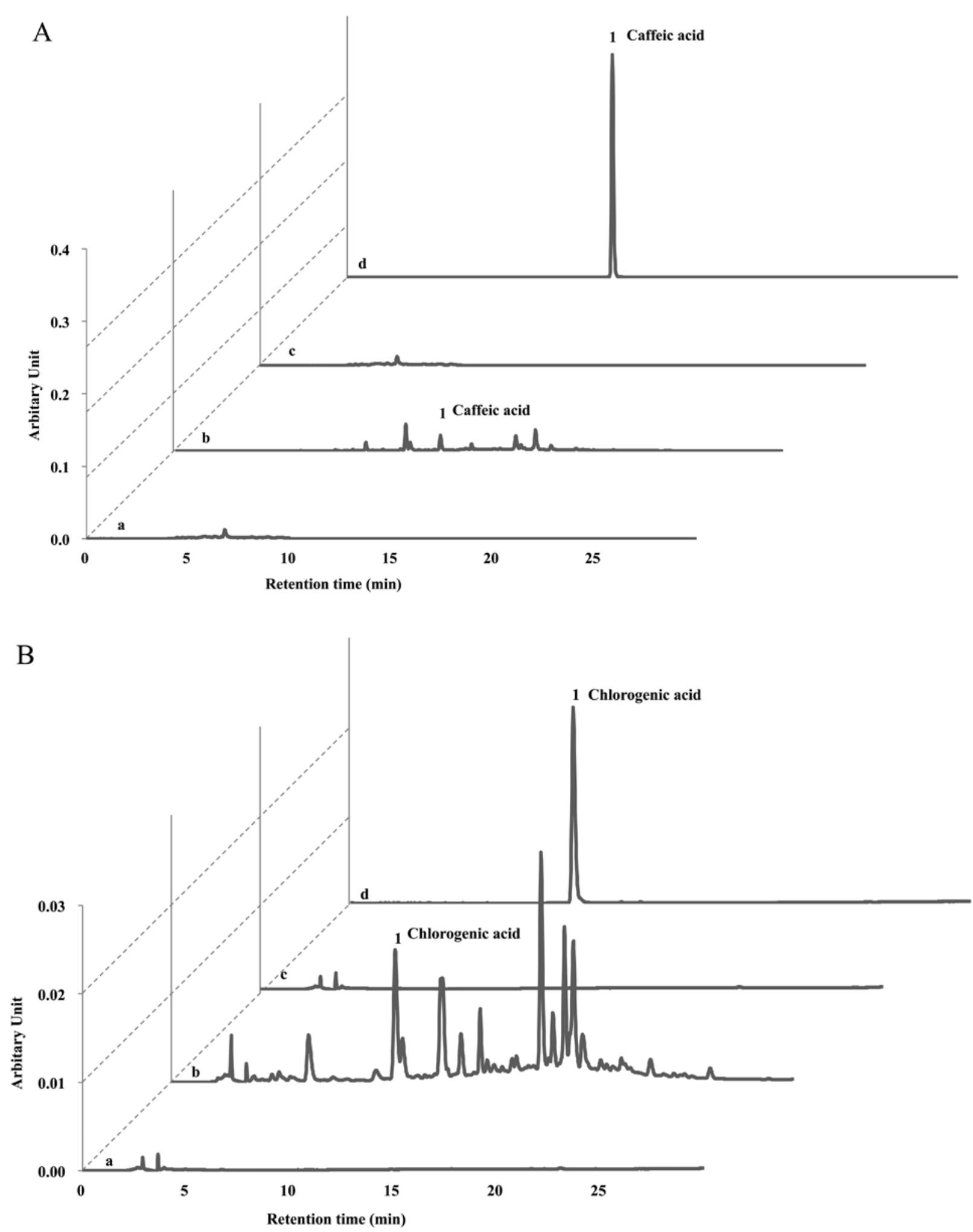

Figure 5. HPLC analysis of Ac and aqueous fraction produced by solid fermentation of Ganoderma lucidum on Artemisia capillaris (afAc/Gl). Sample extract $(10 \mu \mathrm{g} / \mathrm{ml})$ was used for treatment as described in the Materials and methods. Caffeic acid and chlorogenic acid were used as standard materials for HPLC. (A) Measurement of caffeic acid and other compounds in $A c$ or $a f A c / G l$ extract. No peak was observed on the designated retension time period in afAc/Gl extract. (B) Detection of chlorogenic acid on $A c$ or $a f A c / G l$ extract. Lane a, $G l$; lane b, $A c$; lane c, afAc/Gl; lane d, standard (caffeic acid or chlorogenic acid).

the epidermis and dermis from patients with atopic dermatitis, while MMP-9 was not upregulated (33). However, another study demonstrated that MMP-9 was upregulated in a mouse model of DNFB-induced atopic dermatitis, and the IL-31 and T-bet gene were also expressed in the ear epidermis (34). MMP-2 and -12 have been shown to be upregulated in the lung by inspiratory resistive breathing (35). Another study demonstrated that the mRNA levels of MMP-1, MMP-7, MMP-10, 
MMP-14 and MMP-19 were increased independently of COX-2 in monocytes stimulated with LPS (36). Therefore, our results on the MMP regulation pattern imply that $a f A c / G l$ exerts potent effects against atopic dermatitis via the inhibition MMP-2, -7, $-9,-12,-14$ and -19 . Moreover, in a previous study, MMP-9knockout mice exhibited increased levels of IL-4, and IL-13 in lung tissue, as shown by immunohistochemical analysis (37), resulting in an increase in eosinophil and neutrophils counts in bronchioalveolar lavage fluid from MMP-9 knockout mice. MMP-2 knockout mice have been shown to exhibit increased asthmatic symptoms and asphyxiation induced by allergens (38). Moreover, MMP-19-deficient mice have been shown to exhibit exacerbated eosinophilic inflammation in bronchoalveolar lavage fluid and bronchial tissue following allergen challenge (39). Thus, these data indicate that some MMPs are critical to the maintenance of lung tissue, and may protect against asthmatic symptoms. Therefore, MMPs are not only upregulated by allergic inflammation, but also maintaine tissue-specific metabolism in the lungs. Further investigations on the role of MMPs in atopic dermatitis or asthma are warranted in order to develop appropriate curative therapy and/ or of preventive food ingredients.

HPLC analyses of afAc/Gl. The protective effects of the Ac extract (70\% ethanol) against atopic dermatitis have previously been reported (11). Ac contains potent compounds as anti-atopic or inflammatory constituents, such as hyperoside, isoquercetrin, chlorogenic acid, isochlorogenic acid, caffeic acid and scoparone. Therefore, we investigated whether the solid fermentation process can enhance the anti-atopic activity by converting these compounds into more potent ingredients. Surprisingly, we found that the afAc/Gl extract did not show any peaks on the HPLC analysis sheet, although the $A c$ extract contained these compounds (Fig. 5). Since the $G l$ mycelium weight was $<5 \%$ in the afAc/Gl extract and the $G l$ extract showed no activity against atopic dermatitis, we predicted that the active compounds had increased following solid fermentation by converting the compounds, such as chlorogenic acid and caffeic acid. Taken together, these results strongly suggest that isolation and purification of converted compounds is effective, in that converted compound(s) can be used as anti-atopic agent(s), as well as cosmetic ingredient(s) without any toxicity.

In conclusion, the solid fermentation of the Ac extract with $G l$ enhanced the anti-inflammatory effects on atopic dermatitis. In mice with DNFB-induced atopic dermatitis, treatment with $a f A c / G l$ led to a $32 \%$ decrease in ear thickness, and a decrease in eosinophil number of 74\%, which was approximately 2-7-fold more effective than the $A c$ extract without fermentation. Additionally, the numbers of iNOS- and eNOS-positive cells were decreased by treatment with $a f A c / G l$, as shown by immunohistochemical analysis. The afAc/Gl extract was more effective than $A c$ on NO inhibition, as well as on the inhibition of MMP-2, -7, -9, -12, -14 and -19 mRNA expression in RAW264.7 cells. As shown by HPLC analysis, we found that the Ac extract contained caffeic acid, catechin and chlorogenic acid, while the afAc/Gl extract did not. Taken together, we hypothesized that these and other polyphenolic compounds had been changed into novel biomaterial(s) with anti-inflammatory potential by solid fermentation, although we aim to perform further investigations to identify the specific anti-inflammatory ingredient(s) in the
afAc/Gl extract. Importantly however, we demonstrate that solid fermentation is an innovative technology for the enhancement of bioconversion during the processing of natural products.

\section{Acknowledgements}

This study was supported by the Basic Science Research Program through the National Research Foundation of Korea (NRF) funded by the Ministry of Science, ICT and Future Planning (NRF-2014R1A2A2A01006882).

\section{References}

1. Ring J, Alomar A, Bieber T, Deleuran M, Fink-Wagner A, Gelmetti C, Gieler U, Lipozencic J, Luger T, Oranje AP, et al; European Dermatology Forum (EDF); European Academy of Dermatology and Venereology (EADV); European Federation of Allergy (EFA); European Task Force on Atopic Dermatitis (ETFAD); European Society of Pediatric Dermatology (ESPD); Global Allergy and Asthma European Network (GA2LEN): Guidelines for treatment of atopic eczema (atopic dermatitis) part I. J Eur Acad Dermatol Venereol 26: 1045-1060, 2012.

2. Leung DY, Boguniewicz M, Howell MD, Nomura I and Hamid QA: New insights into atopic dermatitis. J Clin Invest 113: 651-657, 2004.

3. Harper JI, Godwin H, Green A, Wilkes LE, Holden NJ, Moffatt M, Cookson WO, Layton G and Chandler S: A study of matrix metalloproteinase expression and activity in atopic dermatitis using a novel skin wash sampling assay for functional biomarker analysis. Br J Dermatol 162: 397-403, 2010.

4. Jeong MS, Choi SE, Kim JY, Kim JS, Kim EJ and Park KH, Lee do I, Joo SS, Lee CS, Bang H, et al: Atopic dermatitis-like skin lesions reduced by topical application and intraperitoneal injection of hirsutenone in NC/Nga mice. Clin Dev Immunol 2010: 618517, 2010.

5. Kim JB, Han AR, Park EY, Kim JY, Cho W, Lee J, Seo EK and Lee KT: Inhibition of LPS-induced iNOS, COX-2 and cytokines expression by poncirin through the NF-kappaB inactivation in RAW 264.7 macrophage cells. Biol Pharm Bull 30: 2345-2351, 2007.

6. Hong SH, Seo SH, Lee JH and Choi BT: The aqueous extract from Artemisia capillaris Thunb. inhibits lipopolysaccharideinduced inflammatory response through preventing NF-kappaB activation in human hepatoma cell line and rat liver. Int $\mathrm{J}$ Mol Med 13: 717-720, 2004.

7. Jang E, Shin MH, Kim KS, Kim Y, Na YC, Woo HJ, Kim Y, Lee JH and Jang HJ: Anti-lipoapoptotic effect of Artemisia capillaris extract on free fatty acids-induced HepG2 cells. BMC Complement Altern Med 14: 253, 2014.

8. Feng G, Wang X, You C, Cheng X, Han Z, Zong L, Zhou C and Zhang M: Antiproliferative potential of Artemisia capillaris polysaccharide against human nasopharyngeal carcinoma cells. Carbohydr Polym 92: 1040-1045, 2013.

9. Lee JH, Park EK, Uhm CS, Chung MS and Kim KH: Inhibition of Helicobacter pylori adhesion to human gastric adenocarcinoma epithelial cells by acidic polysaccharides from Artemisia capillaris and Panax ginseng. Planta Med 70: 615-619, 2004.

10. Lee HI, Seo KO, Yun KW, Kim MJ and Lee MK: Comparative study of the and Artemisia hepatoprotective efficacy of Artemisia iwayomogi capillaris on ethanol-administered mice. J Food Sci 76: T207-T211, 2011.

11. Ha H, Lee H, Seo CS, Lim HS, Lee JK, Lee MY and Shin H: Artemisia capillaris inhibits atopic dermatitis-like skin lesions in Dermatophagoides farinae-sensitized Nc/Nga mice. BMC Complement Altern Med 14: 100, 2014.

12. Martins S, Mussatto SI, Martínez-Avila G, Montañez-Saenz J, Aguilar CN and Teixeira JA: Bioactive phenolic compounds: Production and extraction by solid-state fermentation. A review. Biotechnol Adv 29: 365-373, 2011.

13. Lee IH, Hung YH and Chou CC: Solid-state fermentation with fungi to enhance the antioxidative activity, total phenolic and anthocyanin contents of black bean. Int J Food Microbiol 121: 150-156, 2008.

14. Postemsky P and Curvetto N: Enhancement of wheat grain antioxidant activity by solid state fermentation with Grifola spp. J Med Food 17: 543-549, 2014. 
15. Sánchez-Magaña LM, Cuevas-Rodríguez EO, GutiérrezDorado R, Ayala-Rodríguez AE, Valdez-Ortiz A, Milán-Carrillo J and Reyes-Moreno C: Solid-state bioconversion of chickpea (Cicer arietinum L.) by Rhizopus oligosporus to improve tota phenolic content, antioxidant activity and hypoglycemic functionality. Int J Food Sci Nutr 65: 558-564, 2014.

16. Charlton E: Ethical guidelines for pain research in humans Committee on Ethical Issues of the International Association for the Study of Pain. Pain 63: 277-278, 1995.

17. Shin YK, Son HU, Kim JM, Heo JC, Lee SH and Kim JG: Cinnamomum cassia bark produced by solid-state fermentation with Phellinus baumii has the potential to alleviate atopic dermatitis-related symptoms. Int J Mol Med 35: 187-194, 2015.

18. Bhol KC and Schechter PJ: Topical nanocrystalline silver cream suppresses inflammatory cytokines and induces apoptosis of inflammatory cells in a murine model of allergic contact dermatitis. Br J Dermatol 152: 1235-1242, 2005.

19. Choi EM and Hwang JK: Antiinflammatory, analgesic and antioxidant activities of the fruit of Foeniculum vulgare. Fitoterapia 75: 557-565, 2004.

20. Sato Y, Mukai K, Watanabe S, Goto M and Shimosato Y: The AMeX method. A simplified technique of tissue processing and paraffin embedding with improved preservation of antigens for immunostaining. Am J Pathol 125: 431-435, 1986.

21. Haines DM and Chelack BJ: Technical considerations for developing enzyme immunohistochemical staining procedures on formalin-fixed paraffin-embedded tissues for diagnostic pathology. J Vet Diagn Invest 3: 101-112, 1991.

22. Ständer S and Steinhoff M: Pathophysiology of pruritus in atopic dermatitis: An overview. Exp Dermatol 11: 12-24, 2002.

23. Patruno A, Franceschelli S, Pesce M, Maccallini C,Fantacuzzi M, Speranza L, Ferrone A, De Lutiis MA, Ricciotti E, Amoroso R, et al: Novel aminobenzyl-acetamidine derivative modulate the differential regulation of NOSs in LPS induced inflammatory response: Role of PI3K/Akt pathway. Biochim Biophys Acta 1820: 2095-2104, 2012.

24. Hollá LI, Bucková D, Kuhrová V, Stejskalová A, Francová H Znojil V and Vácha J: Prevalence of endothelial nitric oxide synthase gene polymorphisms in patients with atopic asthma. Clin Exp Allergy 32: 1193-1198, 2002.

25. Yao S, Pandey P, Ljunggren-Rose A and Sriram S: LPS mediated injury to oligodendrocytes is mediated by the activation of nNOS: Relevance to human demyelinating disease. Nitric Oxide 22: 197-204, 2010

26. Moncada S and Higgs EA: Endogenous nitric oxide: Physiology, pathology and clinical relevance. Eur J Clin Invest 21: 361-374, 1991.

27. Jee MK, Im YB, Choi JI and Kang SK: Compensation of cATSCs-derived TGF $\beta 1$ and IL10 expressions was effectively modulated atopic dermatitis. Cell Death Dis 4: e497, 2013.

28. Rowe A, Farrell AM and Bunker CB: Constitutive endothelial and inducible nitric oxide synthase in inflammatory dermatoses. Br J Dermatol 136: 18-23, 1997.
29. Okaniwa N, Sasaki M, Mizushima T, Ogasawara N, Funaki Y, Joh T and Kasugai K: eNOS plays an important role in the regulation of colonic inflammation: A novel therapeutic target and a predictive marker for the prognosis of ulcerative colitis. Free Radic Res 49: 35-44, 2015.

30. De Sanctis GT, MacLean JA, Hamada K, Mehta S, Scott JA, Jiao A, Yandava CN, Kobzik L, Wolyniec WW, Fabian AJ, et al: Contribution of nitric oxide synthases 1,2, and 3 to airway hyperresponsiveness and inflammation in a murine model of asthma. J Exp Med 189: 1621-1630, 1999.

31. Vandenbroucke RE and Libert C: Is there new hope for therapeutic matrix metalloproteinase inhibition? Nat Rev Drug Discov 13: 904-927, 2014.

32. Kim DY, Park BS, Hong GU, Lee BJ, Park JW, Kim SY and Ro JY: Anti-inflammatory effects of the R2 peptide, an inhibitor of transglutaminase 2 , in a mouse model of allergic asthma, induced by ovalbumin. Br J Pharmacol 162: 210-225, 2011.

33. Jin H, Oyoshi MK, Le Y, Bianchi T, Koduru S, Mathias CB, Kumar L, Le Bras S, Young D, Collins M, et al: IL-21R is essential for epicutaneous sensitization and allergic skin inflammation in humans and mice. J Clin Invest 119: 47-60, 2009.

34. Heo JC, Nam DY, Seo MS and Lee SH: Alleviation of atopic dermatitis-related symptoms by Perilla frutescens Britton. Int J Mol Med 28: 733-737, 2011.

35. Toumpanakis D, Noussia O, Sigala I, Litsiou E, Loverdos K, Zacharatos P, Karavana V, Michailidou T, Magkou C, Zhou Z, et al: Inspiratory resistive breathing induces MMP-9 and MMP-12 expression in the lung. Am J Physiol Lung Cell Mol Physiol 308: L683-L692, 2015.

36. Reel B, Sala-Newby GB, Huang WC and Newby AC: Diverse patterns of cyclooxygenase-independent metalloproteinase gene regulation in human monocytes. Br J Pharmacol 163: 1679-1690, 2011.

37. Corry DB, Kiss A, Song LZ, Song L, Xu J, Lee SH, Werb Z and Kheradmand F: Overlapping and independent contributions of MMP2 and MMP9 to lung allergic inflammatory cell egression through decreased CC chemokines. FASEB J 18: 995-997, 2004.

38. Corry DB, Rishi K, Kanellis J, Kiss A, Song Lz LZ, Xu J, Feng L, Werb Z and Kheradmand F: Decreased allergic lung inflammatory cell egression and increased susceptibility to asphyxiation in MMP2-deficiency. Nat Immunol 3: 347-353, 2002.

39. Gueders MM, Hirst SJ, Quesada-Calvo F, Paulissen G, Hacha J, Gilles C, Gosset P, Louis R, Foidart JM, Lopez-Otin C, et al: Matrix metalloproteinase-19 deficiency promotes tenascin- $\mathrm{C}$ accumulation and allergen-induced airway inflammation. Am J Respir Cell Mol Biol 43: 286-295, 2010. 